\title{
Lifetime and Energy Efficiency Improvement Techniques for Hierarchical Networks
}

\author{
Suhas A. R., Manoj Priyatham M.
}

\begin{abstract}
The detection points are the detection points in the space of network. The properties of detection points include cost effective materials and longer battery capacity. WSN can span variety of applications like sensing of data related to environment entities, detection of enemy vehicles. Lifetime ratio defines the efficiency of the WSN network operation. There are multiple techniques which can help in improvement of Network Lifetime (NL) spanning from transmission nature, data connections, formation of System and time scheduling. This paper provides the analysis of how energy consumption happens and its effect on lifetime ratio. $\mathrm{LEACH}$ and $\mathrm{CHEF}$ algorithms responsible for hierarchical kind of routing are discussed in detail with simulation results. The parameters used for comparison includes delay, hops, consumption of energy. Non-Hole detection points, Hole detection points, Non-Hole to Hole Ratio, residual energy, routing overhead and throughput.
\end{abstract}

Keywords- Hole, Chance Computation, System Formation.

\section{INTRODUCTION}

The class label of Wireless Sensor Network (WSN) can be done into single or multiple system networks with each system bounded by area limits. In a multi system based network, the entire detection points are divided into multiple independent areas. For a single System network all detection points are placed in a single area. For effective communication a special detection point is chosen from the System and it is referred as System head [1]. The Received-signal-strength (RSS) is computed for the various sensors in order inject the detection points in the network graph. The estimation of power, determination of location along with direction of sources is done by various algorithms [2].

The flexibility ratio along with easy access is better for WSN as compared to a connected wired network. The channel can be selected in a sequential manner applied on the subset of sensors or in a randomized manner applied on subset of sensors [3].

The health issues faced by the community are increasing in the exponential manner. Due to lack of doctors in the remote villages, patient suffers a lot. The health care factors include sensitivity of time, critical nature of data. The Wireless Medical Sensor Networks (WMSNs) are used for such applications [4].

When the rate of transmission of data packets towards a lot is higher than detection point can handle will cause packet drops, data retransmissions and collision of data [5].

Revised Manuscript Received on December 15, 2019

Suhas A. R., Research Scholar, Dept. of ECE, A P S College of Engineering, Bengaluru, India. E-mail: suhas.ar8@gmail.com

Dr. Manoj Priyatham M., Professor, Dept. of ECE, A P S College of Engineering, Bengaluru, India. E-mail: drmmp777@gmail.com
The cat swarm optimization (CSO) is combined along with exponential weighted moving average. Ant colony method is used to select the System head. The search optimization techniques like fuzzy, penguin search are combined with ACO to achieve better path efficiency. During this process the nest detection point is selected based on multiple parameters [6].

Few areas in which WSN is used is included in fig.1.

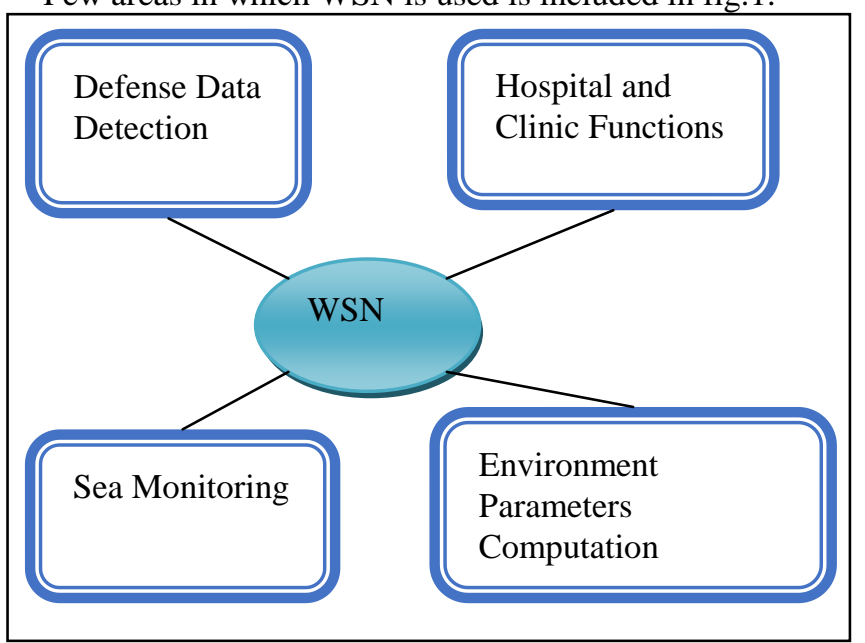

Fig. 1: WSN application nature

Fig. 1 shows the WSN applications like Defense, Analysis of health, and monitoring nature of sea and data capture for environment. The major classification of WSN includes Single System and Multi System Network.

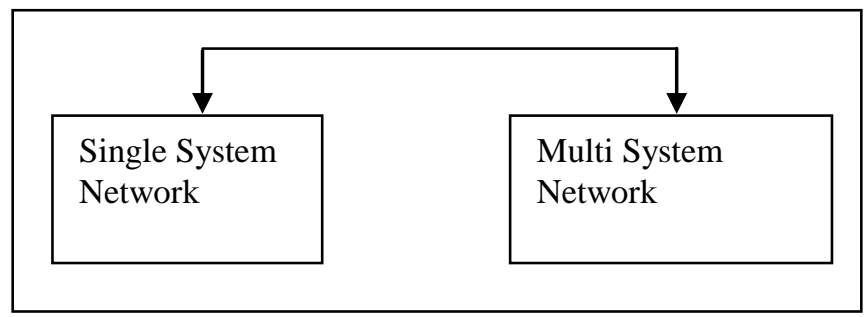

Fig. 2: Broader Classification of WSN Network

The networks are divided into Single System and Multi System. Single System will have detection points spread in a single bounded area. For a Multi System Network the detection points are spread across multiple independent bounded areas. The Single System based system is provided in the fig. 3 . 


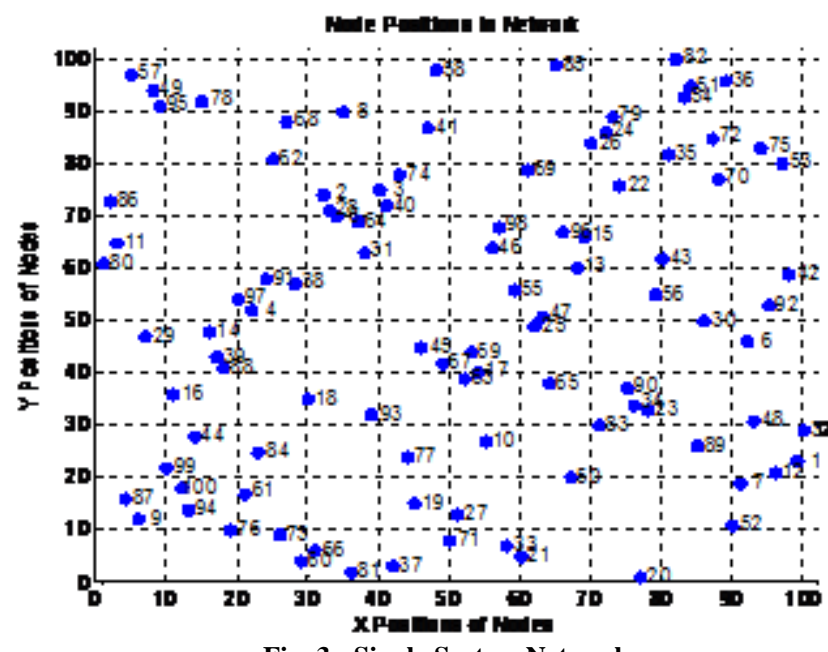

Fig. 3: Single System Network unique detection points are placed in $(1,100) \times$ limits and $(1,100)$ y limits. Detection point 9 in at the position $(9,11)$, Detection point 52 is at the position $(90,10)$ and Detection point is present at $(4,68)$. In a similar fashion all the detection points in the network will be at unique positions in the WSN network. The multi system based network is formed by combining a set of single system networks.

Each detection point follows limit theorem of boundness between $\mathrm{x}$ position and y position as well as it will have the detection points with their own unique ids in the network.

If the two detection points have the same System, then communication will happen in the direct manner. If the two detection points belong to different System, then communication happens with the help of System head. The multi System based network is depicted in in fig. 4 .

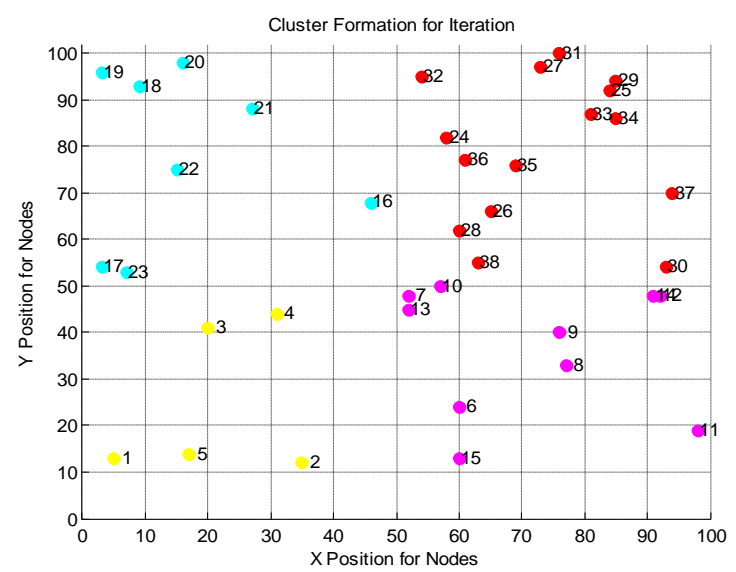

Fig. 4: Multi system Network

Fig. 4 shows the multi system network. As shown in the fig. 4 there are four different color detection points. In the system1 there are set of detection points which follow limit theorem of edges $(1,50)$ and $(1,50)$. System 2 contains 10 detection points within the limit theorem of edges $(51,100)$ and $(1,50)$. System 3 contains 8 detection points within the limit theorem of edges $(1,50)$ and $(51,100)$ and System 4 contains 15 detection points within the limit theorem of edges $(51,100)$ and $(51,100)$.
Fig. 3 shows the single system nature for network in which the

Section II will provide the information about dissipation of energy parameter. Section III will define the Non-Hole to Hole ratio. Section IV will provide overview of LEACH and $\mathrm{CHEF}$ method. Section $\mathrm{V}$ will provide the simulation results for existing $\mathrm{LEACH}$ and $\mathrm{CHEF}$ method.

\section{ENERGY DISIPATION PROCESS}

The energy dissipation [7]-[10] for the transmission of information is given by the equation.

$$
E_{c}=2 * E_{t x}+E_{g e n} d^{\delta}
$$

If a link is to be established, then Etx is the energy required for link formation. Egen is the energy required for control packet sending between detection points, $\mathrm{d}$ is the distance between the participating detection point and delta is the dependency factor based on temperature range.

The detection points are divided into hole detection points and non-hole detection points. Hole Detection points are set of detection points whose residual energy will be less than threshold during network formation and Non-Hole detection points are the detection points whose residual energy is higher than threshold or equal to it. If IBE is the energy during network detection point values then threshold would be (IBE/4)

\section{Hole}

\section{Non-Hole}
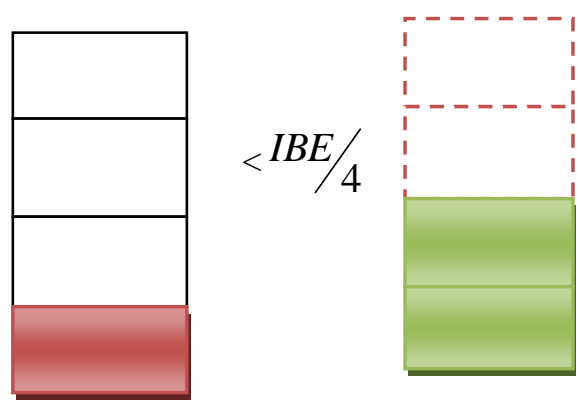

$>=I B E / 4$

Fig. 5: Hole and Non-Hole Energy in the Network

Fig. 5 shows the Hole and Non-Hole detection points. For an bounded theorem of $100 * 100$ and set of 10 detection points the first value for energy is defined in table 1 .

Table 1 First Energy of Detection Points

\begin{tabular}{|c|c|}
\hline Detection Point & Residual Energy (J) \\
\hline 1 & 1000 \\
\hline 2 & 1000 \\
\hline 3 & 1000 \\
\hline 4 & 1000 \\
\hline 5 & 1000 \\
\hline 6 & 1000 \\
\hline 7 & 1000 \\
\hline 8 & 1000 \\
\hline 9 & 1000 \\
\hline 10 & 1000 \\
\hline
\end{tabular}

Table 1 shows the energy of the detection points, as shown in the table all the detection points are filled with same value of parameter $1000 \mathrm{~J}$. 
The value IBEv/4 = 1000/4 = $250 \mathrm{~J}$

The data sending links are defined as below.

$6 \rightarrow 8 \rightarrow 9 \rightarrow 10$

The data sending links contains the set of detection points Detection Point 6, Detection Point 8, Detection Point 9 and Detection Point 10. The distance between the detection points is assumed to be as defined in table 2 .

Table 2: Detection Points between Detection points values

\begin{tabular}{|c|c|}
\hline $\begin{array}{c}\text { Link Between Two Detection } \\
\text { points }\end{array}$ & $\begin{array}{c}\text { Detection Point From } \\
\text { Topology }\end{array}$ \\
\hline $6 \rightarrow 8$ & 22.24 \\
\hline $8 \rightarrow 9$ & 47.56 \\
\hline $9 \rightarrow 10$ & 36.56 \\
\hline
\end{tabular}

Since Detection Point 6, Detection Point 8, Detection Point 9 and Detection Point 10 are present in the data link path and hence energy dissipation happens.

The Updated Energy of Detection points is defined using the following equation.

UEdetection point $=$ CEdetection point - Ec

Where,

CEdetection point $=$ current energy parameter of detection point

UEdetection point $=$ update energy parameter of detection point

$\mathrm{Ec}=$ Energy dissipation

The updated dissipation of energy of Detection point 8 after the link is established between $6 \rightarrow 8$

UE6 $=$ CE6- $\operatorname{Ec}(6,8)=1000-\left(2 * 20+10^{*} \mathrm{~d}(6,8)^{\wedge} 0.5\right)=1000$ $-\left(2 * 20+10 * 22.24^{\wedge} 0.5\right)=912.8407 \mathrm{~J}$

The detection points energy parameter after the link formation is defined as given in Table 3 .

Table 3: Updated Detection Points Parameter Levels

\begin{tabular}{|l|l|}
\hline Detection Point & Residual Energy (J) \\
\hline 1 & 1000 \\
\hline 2 & 1000 \\
\hline 3 & 1000 \\
\hline 4 & 1000 \\
\hline 5 & 1000 \\
\hline 6 & $912.8407 \mathrm{~J}$ \\
\hline 7 & 1000 \\
\hline 8 & $891.0362 \mathrm{~J}$ \\
\hline 9 & $899.5351 \mathrm{~J}$ \\
\hline 10 & 1000 \\
\hline
\end{tabular}

Residual Energy of the detection points after path is discovered then updated energy of the detection points is found out. Like this process is repeated until 100 iterations are completed then residual energy is kept on decreasing for participating detection points and suppose after that the remaining energy of the detection points is as defined in Table 4.
Detection point 6 and Detection point 8 are hole Detection points and remaining detection points will be Detection point 2, Detection point 3, Detection point 4 , Detection point 5, Detection point 7, Detection point 9 and Detection point 10 will be non-hole detection points.

Table 4: Detection Point Energy After 100 iterations

\begin{tabular}{|l|l|}
\hline Detection Point & Residual Energy \\
\hline 1 & 1000 \\
\hline 2 & 1000 \\
\hline 3 & 1000 \\
\hline 4 & 1000 \\
\hline 5 & 1000 \\
\hline 6 & $147.89 \mathrm{~J}$ \\
\hline 7 & 1000 \\
\hline 8 & 153.89 \\
\hline 9 & 654.89 \\
\hline 10 & 1000 \\
\hline
\end{tabular}

\section{NON-HOLE TO HOLE RATIO}

The Non-Hole to Hole ratio is defined as the ratio of Non-Hole versus the Hole detection points. The Lifetime Ratio (LR) will be defined with the following value

$$
L R=\frac{\text { count of nodes which are Non }- \text { Hole nodes }}{\text { count of nodes which are Hole nodes }}
$$

When the same detection points participate again and again in a repeated fashion then a point is reached at which the energy is below threshold and hence decreases the value of LR.

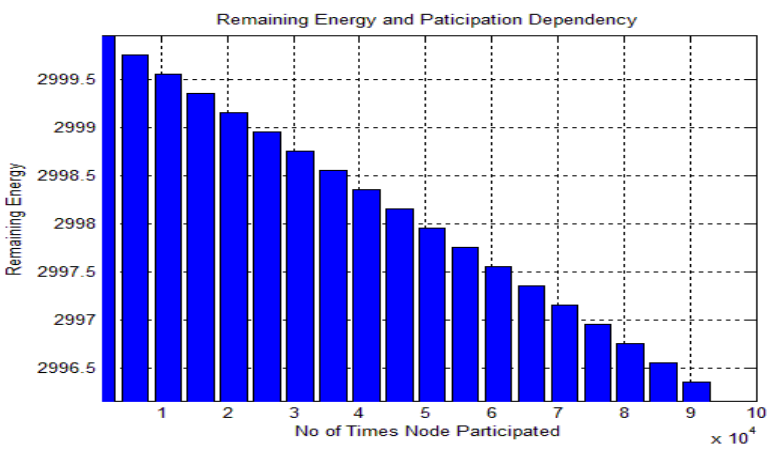

Fig6: HOLE/NON-HOLE PARAMETRIC MEASURE

Fig. 6 shows the HOLE/NON-HOLE parametric measure in which the detection point loses its energy levels based on participating probability. From the fig before first iteration it has a value $3000 \mathrm{~J}$ as the number of times detection point participated then energy of detection points reduces.

\section{LIFETIME DEFINITIONS}

The specific objective of the network is to maintain an optimized working of WSN [11]. The reduction of energy consumption will depend on transmission energy, 
amplification energy, distance value and then attenuation factor. The number of detection points which loses there energy and become a hole detection point after some period of time ' $\mathrm{T}$ ' then it is defined as the lifetime ratio [12]-[15] as summarized in Table 5.

Table 5: Network Lifetime Definition

\begin{tabular}{|c|c|}
\hline $\begin{array}{l}\text { NON-HOLE } \\
\text { to HOLE } \\
\text { RATIO(LR) }\end{array}$ & Definition \\
\hline LR-1 & SNR satisfaction is well above threshold [16] \\
\hline LR-2 & $\begin{array}{l}\text { The amount of time until which at least a single } \\
\text { detection point exists which can maintain the } \\
\text { connectivity [17] }\end{array}$ \\
\hline LR-3 & $\begin{array}{l}\text { The amount of time after which zone leader (ZL) } \\
\text { loses its energy completely [18] }\end{array}$ \\
\hline LR-4 & $\begin{array}{l}\text { When the packet delivery ratio becomes below a } \\
\text { certain threshold [19] }\end{array}$ \\
\hline
\end{tabular}

\section{ALGORITHMS}

\section{A. Detection Point Location Strategy}

Detection point location strategy is responsible for location determination of detection points in the network. The input will be number of detection points and end points of area. The output will be unique detection point id, along with Cartesian co-ordinates of detection point. The Detection Point Location Strategy is summarized in fig. 7.

\section{ALGORITHM: DETECTION POINT STARTEGY}

Input: Number of Detection Points, End points of area Output: Node ID, X position, Y position Algorithm

1. Number of Detection Points, End points of a zone

2. Start from the value of $i=1$ till the end of the number of Detection Point (DP)

3. Generate the Node Id is ' $\mathrm{i}$ '

4. Generate the $\mathrm{x}$ position of node between $\mathrm{xmin}$ to $\mathrm{xmax}$ as Xposition

5. Generate the y position of node between ymin to ymax as Yposition

6. Form the triplet (NodeId,Xposition, Yposition)

7. Save the value in the ith row of Node Placement Matrix

8. Increment the value of $i$

9. Repeat the process until complete node placement.

Fig. 7: Detection point Placement Algorithm

The Multi System formation is responsible for formation of detection points across the network. Each system will have the set of detection points. The Multi System is summarized in the Multi System Formation.
ALGORITHM: Multi System Algorithm

Input: Number of Systems, End Points of System, Number of Detection Points in each System Output: Multi System Formation

Algorithm

1. Number of Systems, End points of System and Number of Detection Points in each System

2. Start from $1^{\text {st }}$ node till the number of Detection Points in the network

3. Find the number of Detection Points required for the ith System

4. Find the end points of ith System

5. Execute the Detection Point Strategy and generate the Node Placement Matrix.

6. Generate the System Id as i

7. Increment the value of $i$

8. Repeat process until all System Formation is completed

Fig. 8: Multi System Formation

\section{B. $\mathrm{LEACH}$}

LEACH algorithm will first perform Systeming of detection points across the areas. System head is selected based on computation of probability. From each of the System one of the detection points is elected based on probability value. The communication within the System happens between two detection points. When the communication happens between two Systems then it has to go through System head. The algorithm will be defined as below.

\section{ALGORITHM: LEACH ALGORITHM}

Input: Initiator Detection Point, Control Center Detection Point

Output: Path between Initiator Detection Point, to Control Center Detection Point

Details

1. Initiator Detection Point, Control Center Detection Point will act as input

2. If the Initiator Detection Point, and Control Center Detection Point are within the same System, then detection point will communicate with other detection point directly

3. If the source Detection Point and destination Detection Point belong to different Systems, then send the packet to System head of the initiator cluster

4. From the initiator System, the System head will send the communication to base station.

5. The base station will communicate to each other Systems sequentially

6. Repeat process until destination Detection Point is rearhed

Fig. 9: LEACH Algorithm 
Fig. 9 shows the various steps involved in LEACH algorithm. The System head will be defined based on randomized probability and during each round the System head will vary.

\section{Chef}

CHEF algorithm will optimize the routing process. The CHEF algorithm will elect the System head based on residual energy and distance based on chance factor. The chance factor is computed based on Table 5. Table 5 shows the computation of chance. Higher than chance higher is the probability of detection point getting elected as System head. Chance is higher if residual energy is high and lesser distance. The route path is found out based on LEACH method even in CHEF process. The CHEF based System head selection is summarized in fig. 10 .

In the truth table $\mathrm{L}$ indicates LOW, $\mathrm{F}$ indicates FAR, M indicates MEDIUM, $\mathrm{H}$ indicates $\mathrm{HIGH}$ and $\mathrm{N}$ indicates NEAR.

\begin{tabular}{llll}
\hline ALGORITHM: & CHEF & SYSTEM & HEAD \\
ALGORITHM & & & \\
\hline
\end{tabular}

Input: Nodes Placement Matrix

Output: Single Detection Point acting as System Head Details

1. Find the Detection Points of Specific System

2. Find the residual energy for all Detection Points in the network

3. Find the distance for all Detection Points in the network

4. Compute the complete factor based on residual energy and distance which is based on fuzzy truth table

5. Compute the chance variable and which has highest chance will be selected for the System head.

Fig. 10: CHEF System Head Algorithm

The trust table for selection of System Head for CHEF algorithm is defined in the truth table 5.

Table 5: Trust Table Computation

\begin{tabular}{|c|c|c|c|}
\hline SI No & $\begin{array}{c}\text { Remaining } \\
\text { Energy of } \\
\text { Detection } \\
\text { Point }\end{array}$ & $\begin{array}{c}\text { Distance } \\
\text { with } \\
\text { respect to } \\
\text { Base } \\
\text { Station }\end{array}$ & $\begin{array}{c}\text { Selection } \\
\text { Factor }\end{array}$ \\
\hline 1 & $\mathrm{~L}$ & $\mathrm{~F}$ & V.SMALL \\
\hline 2 & $\mathrm{~L}$ & $\mathrm{M}$ & R.SMALL \\
\hline 3 & $\mathrm{~L}$ & $\mathrm{~N}$ & SMALL \\
\hline 4 & $\mathrm{M}$ & $\mathrm{F}$ & S.MEDIUM \\
\hline 5 & $\mathrm{M}$ & $\mathrm{M}$ & MEDIUM \\
\hline 6 & $\mathrm{M}$ & $\mathrm{N}$ & V.MEDIUM \\
\hline 7 & $\mathrm{H}$ & $\mathrm{F}$ & S.LARGE \\
\hline 8 & $\mathrm{H}$ & $\mathrm{M}$ & LARGE \\
\hline 9 & $\mathrm{H}$ & $\mathrm{N}$ & V.LARGE \\
\hline
\end{tabular}

Table 6: Simulation Input LEACH

\begin{tabular}{|c|c|}
\hline Parameter Name & Parameter Value \\
\hline $\begin{array}{l}\text { Number of Detection } \\
\text { points }\end{array}$ & 100 \\
\hline Area & $100 * 100$ \\
\hline Coverage Area & $40 \mathrm{~m}$ \\
\hline Transmission Energy & $20 \mathrm{~mJ}$ \\
\hline Generation Energy & $10 \mathrm{~mJ}$ \\
\hline Environment Factor & 0.5 \\
\hline First Energy & $1000 \mathrm{~mJ}$ \\
\hline Threshold Count & 4 \\
\hline Source Detection point & 8 \\
\hline $\begin{array}{ll}\begin{array}{l}\text { Destination } \\
\text { point }\end{array} & \text { Detection } \\
\end{array}$ & 33 \\
\hline End points for System 1 & $\begin{array}{l}X \min =1 X \max =50 \\
Y \min =1 \quad Y \max =50\end{array}$ \\
\hline End points for System 2 & $\begin{array}{l}X \min =51 X \max =100 \\
Y \min =1 \mathrm{Ymax}=50\end{array}$ \\
\hline End points for System 3 & $\begin{array}{l}X \min =1 \quad X \max =50 \\
Y \min =51 \quad Y \max =100\end{array}$ \\
\hline End points for System 4 & $\begin{array}{l}X \min =51 \quad X \max =100 \\
Y \min =51 \quad Y \max =100\end{array}$ \\
\hline $\begin{array}{l}\text { Number of Detection } \\
\text { points for System } 1\end{array}$ & 10 \\
\hline $\begin{array}{l}\text { Number of Detection } \\
\text { points for System } 2\end{array}$ & 10 \\
\hline $\begin{array}{l}\text { Number of Detection } \\
\text { points for System3 }\end{array}$ & 10 \\
\hline $\begin{array}{l}\text { Number of Detection } \\
\text { points for System } 4\end{array}$ & 10 \\
\hline
\end{tabular}

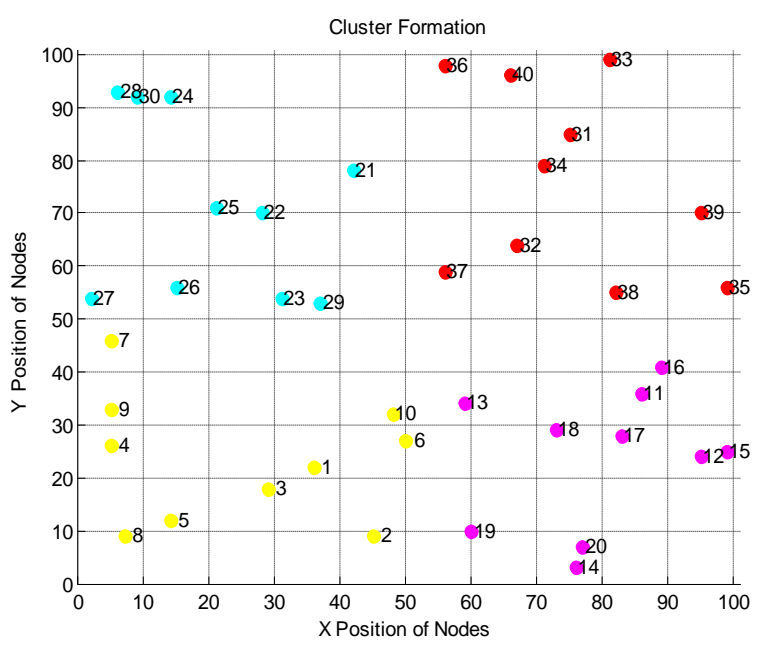

Fig. 11: System Formation

Fig. 11 shows the System formation. As shown in the fig. 11 there are four Systems. Each System has unique set of detection points spread in it. 
Four Systems totally have 40 detection points. System 1 has 10 detection points with a value $\{$ Detection point 1 , Detection point 2, Detection point 3, Detection point 4 , Detection point 5. Detection point 6, Detection point 7, Detection point 8, Detection point 9 and Detection point 10$\}$. In the same way remaining 3 Systems have their detection points in the System.

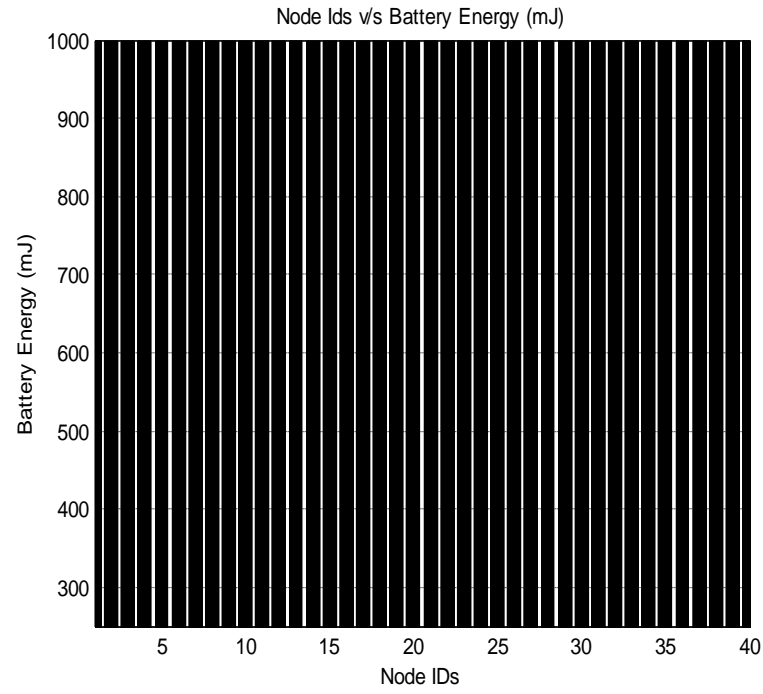

Fig. 12: Detection points Initialization for Network

Fig. 12 shows the initialization of network. As shown in the fig all the detection points have $1000 \mathrm{~J}$ of energy level. All the 40 detection points in the network have been provided with same amount of energy of $1000 \mathrm{~J}$.

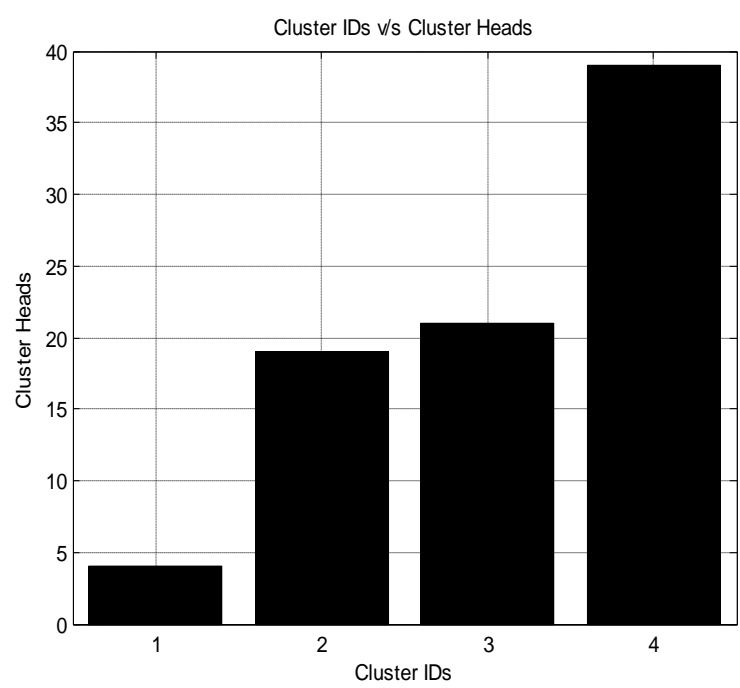

Fig. 13: System Ids versus System Heads

Fig. 13 shows the $\mathrm{x}$ axis as the System ids and it varies from System 1 to System 4. For the System 1 the System Head is selected as Detection point 4 for System 1, System Head is selected as Detection point 19, System Head will have selected as Detection point 21 for System 3 and Detection point 38 will act as the System Head for System 4.

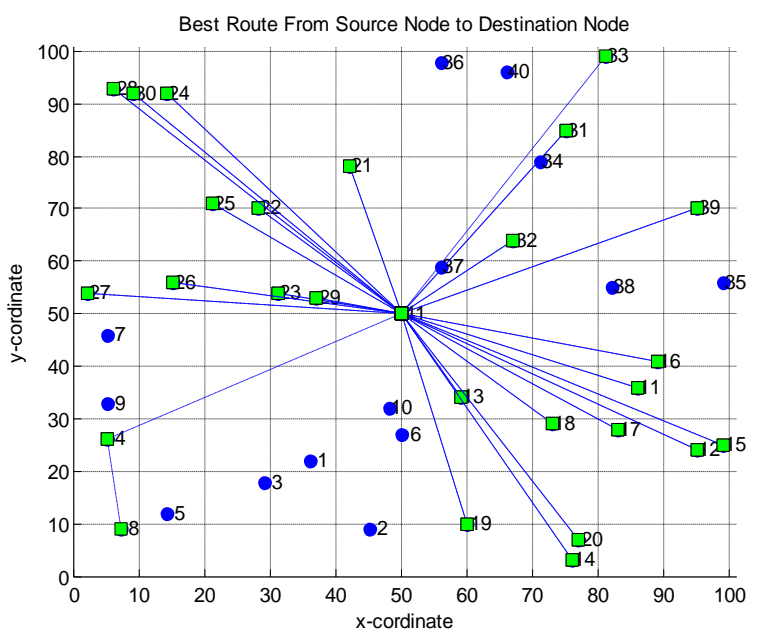

Fig. 14: Best Route from Source Detection point to Destination Detection point

The best route from source detection point to destination detection point is found out by taking Detection point 8 as the initiator detection point and Detection point 33 as the destination detection point. The communication happens from Detection point 8 to Detection point 4 which is the System head of initiating System, Detection point 4 communicates to base station and the base station scans the remaining Systems until destination detection point is reached.

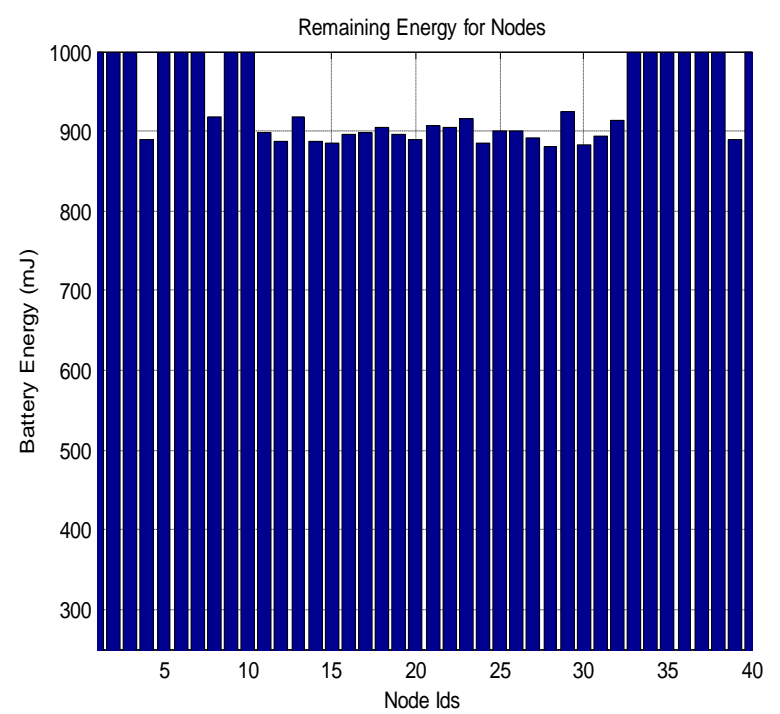

Fig. 15: Residual Energy for Detection points

Fig. 15 shows the residual energy for detection points. As shown in the Fig 15 all the detection points which are participating in the network will reduce their remaining energy.

\section{B. CHEF Algorithm}

The CHEF algorithm will be used to find the path between two detection points in the network and for the same CHEF algorithm the communication happens between initiator detection point Detection point 6 to Destination Detection point i.e Detection point 36. 
Table 7: Simulation Input CHEF

\begin{tabular}{|c|c|}
\hline Parameter Name & Parameter Value \\
\hline Number of Detection points & 100 \\
\hline Area & $100 * 100$ \\
\hline Coverage Area & $40 \mathrm{~m}$ \\
\hline Transmission Energy & $20 \mathrm{~mJ}$ \\
\hline Generation Energy & $10 \mathrm{~mJ}$ \\
\hline Environment Factor & 0.5 \\
\hline First Energy & $1000 \mathrm{~mJ}$ \\
\hline Source Detection point & 6 \\
\hline Destination Detection point & 36 \\
\hline End points for System1 & $\begin{array}{l}X \min =1 \quad X \max =50 \\
Y \min =1 \quad Y \max =50\end{array}$ \\
\hline End points for System 2 & $\begin{array}{l}X \min =51 \quad X \max =100 \\
Y \min =1 \quad Y \max =50\end{array}$ \\
\hline End points for System3 & $\begin{array}{l}X \min =1 \quad X \max =50 \\
Y \min =51 Y \max =100\end{array}$ \\
\hline End points for System 4 & $\begin{array}{l}X \min =51 X \max =100 \\
Y \min =51 Y \max =100\end{array}$ \\
\hline $\begin{array}{l}\text { Number of Detection points } \\
\text { for System1 }\end{array}$ & 10 \\
\hline $\begin{array}{l}\text { Number of Detection points } \\
\text { for System } 2\end{array}$ & 10 \\
\hline $\begin{array}{l}\text { Number of Detection points } \\
\text { for System3 }\end{array}$ & 10 \\
\hline $\begin{array}{l}\text { Number of Detection points } \\
\text { for System } 4\end{array}$ & 10 \\
\hline
\end{tabular}

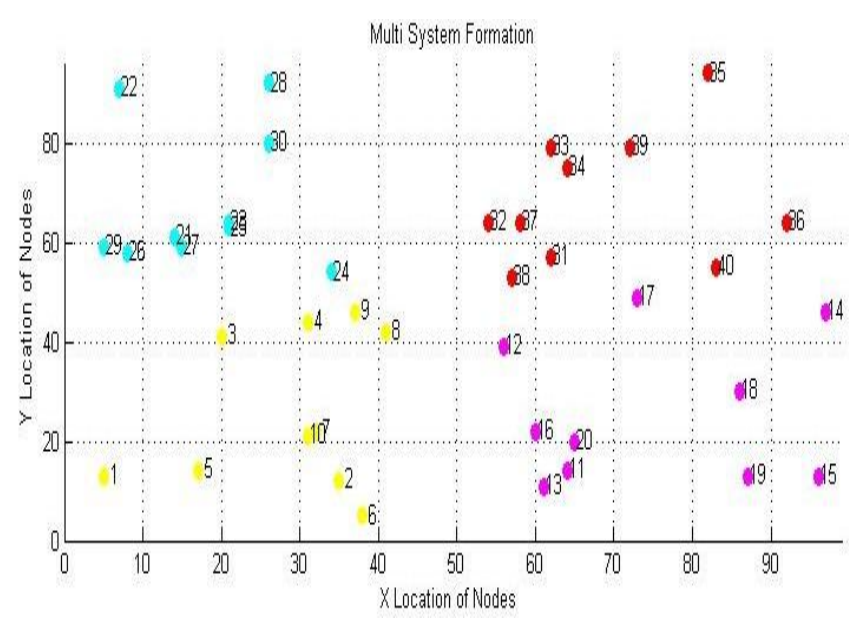

Fig. 16: System Formation for Network

Fig. 16 shows the System formation. As shown in the fig. 11 there are four Systems. Each System has unique set of detection points spread in it. Four Systems totally have 40 detection points. System 1 has 10 detection points with a value \{ Detection point 1, Detection point 2, Detection point 3 , Detection point 4 , Detection point 5 . Detection point 6 , Detection point 7, Detection point 8, Detection point 9 and Detection point 10$\}$. In the same way remaining 3 Systems have their detection points in the System.

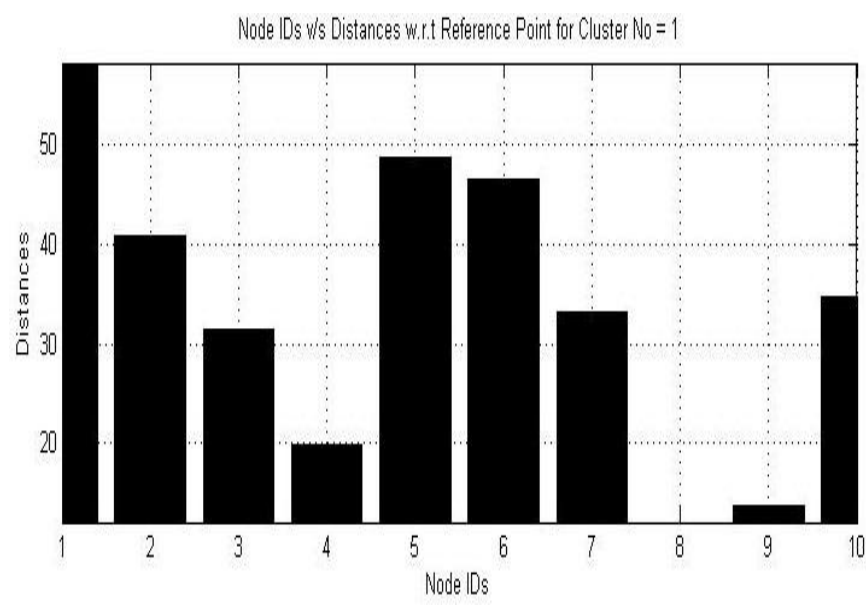

Fig. 17: Detection point Position v/s Distances with respect to System No1

Fig. 17 shows the $\mathrm{x}$-axis which informs about various detection points in the network ranging from Detection point 1 to Detection point 10 and $y$-axis indicates the distance of detection point with respect to reference point in the network. As shown in the fig. 16 Detection point 5 is placed at a distance of $4 \mathrm{~m}$ with respect to System 1 . In a similar fashion various other detection points are placed in the network.

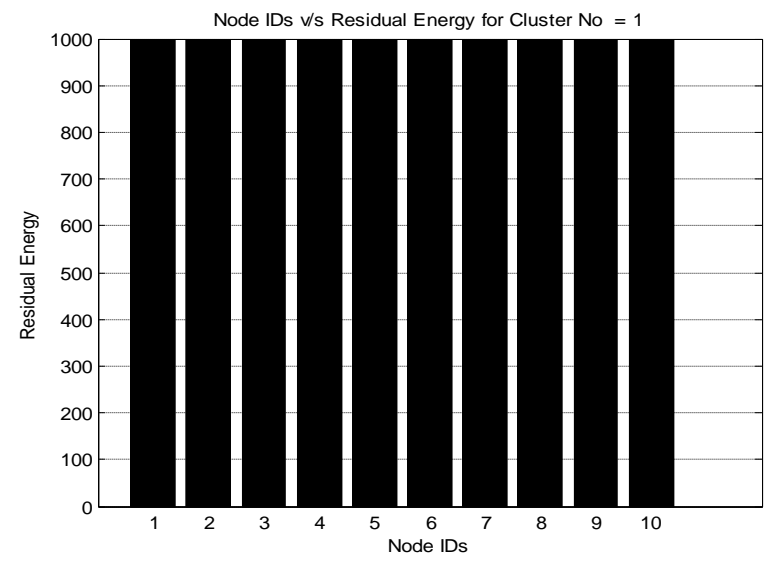

Fig. 18: Detection point Battery Levels

Fig. 18 shows the battery levels for all the detection points in the network. As shown in the fig. 18 all the detection points from Detection point 1 to Detection point 10 have been initialized to same value of $1000 \mathrm{~J}$. By using the truth table defined in Table 5 the System head is selected.

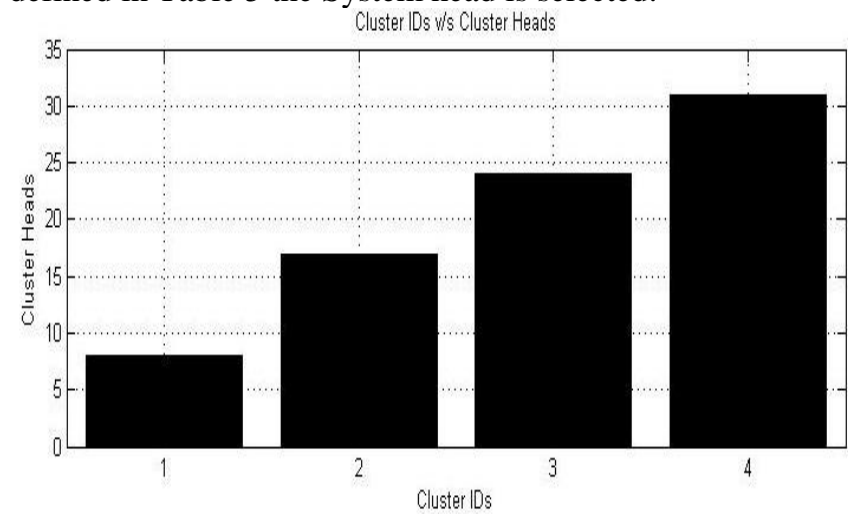

Fig. 19: System Head Selection

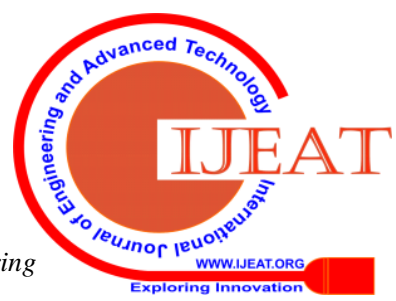


Fig. 19 shows the System head selection. The $\mathrm{X}$-axis indicates the detection point ids present in the network. The $\mathrm{Y}$-axis is the System head present in the network. For the System 1 Detection point 8 is the System Head, For the System 2 the selected System head is Detection point 17, For the System 3 the selected System head is Detection point 24 and for the final System i.e System 4 the selected System head is Detection point 31 .

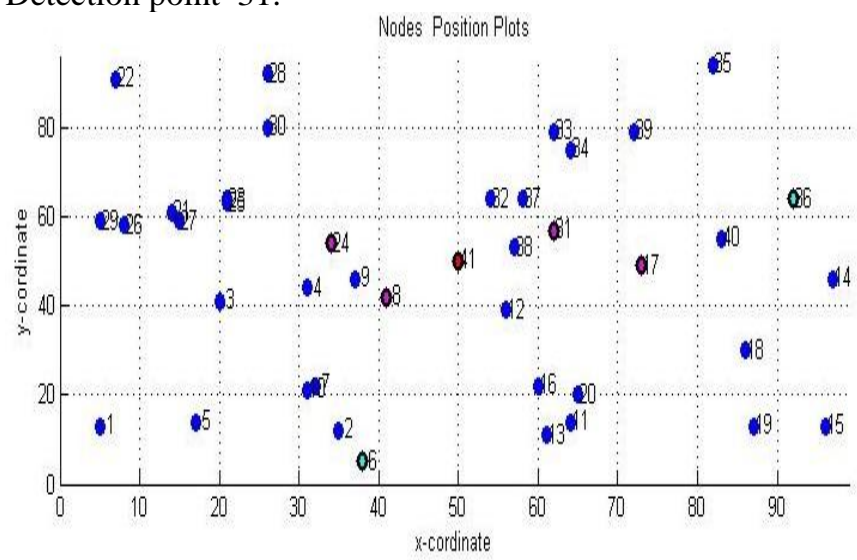

Fig. 20: Detection point Placement for System Heads

Fig. 20 shows the detection point placement for System heads. As shown in the fig. 20 the red color ones are the System heads. The System heads are Detection point 8, Detection point 17, Detection point 24 and Detection point 31.

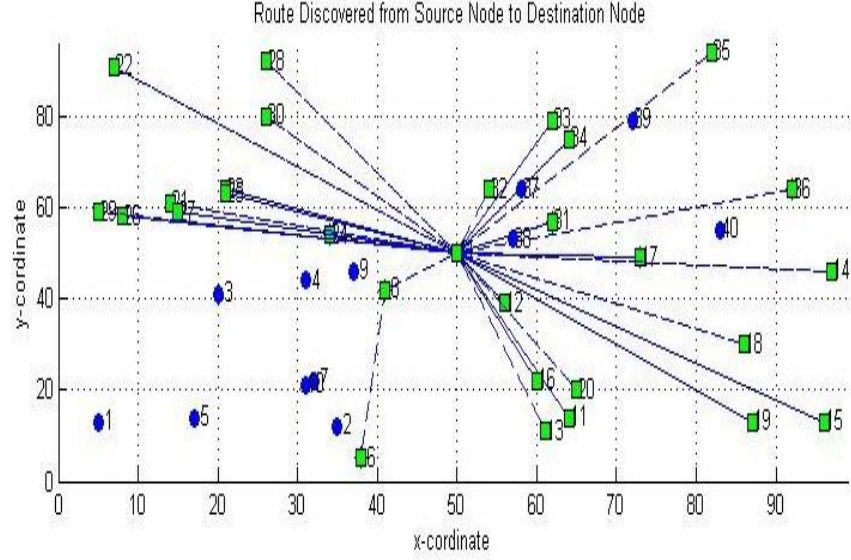

Fig. 21: Route discovered using CHEF Method

Fig. 21 shows the end to end route path defined by the CHEF algorithm. As shown in the fig. 21 the packet is send from initiator Detection point 6 to Detection point 8 and then to base station, from the base station every System is scanned in a sequential fashion until all Systems are completed.

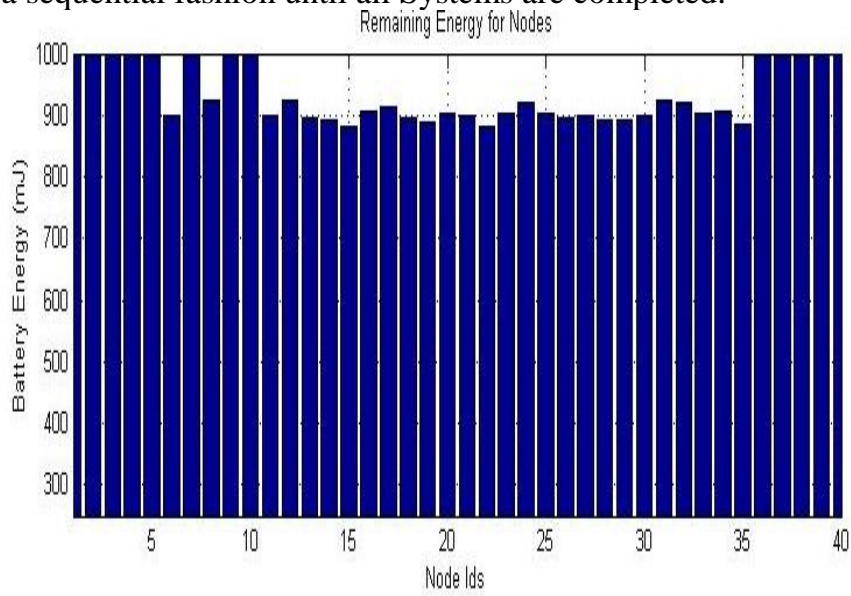

Fig 20: Residual Energy for Detection points
Fig 20 shows the residual energy for detection points. As shown in the Fig 20 for all the participating detection points the energy level for the detection points come down based on distance with respect to other detection points in the path.

\section{COMPARISON}

The comparison between LEACH and CHEF is performed on the Multi-System Network with each System having a set of detection points. The various parameters involved in the comparison are delay, hops, energy consumed. Number of Non-Hole detection points, number of Hole detection points, lifetime ratio, residual energy, overhead and throughput

The simulation input for the comparison results can be defined as below

Table 8: Comparison of All Algorithms

\begin{tabular}{|l|l|}
\hline Parameter Name & Parameter Value \\
\hline Number of Detection points & 100 \\
\hline Area & $100 * 100$ \\
\hline Coverage Area & $40 \mathrm{~m}$ \\
\hline Transmission Energy & $20 \mathrm{~mJ}$ \\
\hline Generation Energy & $10 \mathrm{~mJ}$ \\
\hline Environment Factor & 0.5 \\
\hline First Energy & $1000 \mathrm{~mJ}$ \\
\hline Source Detection point & 1 \\
\hline Destination Detection point & 38 \\
\hline End points for System1 & $\begin{array}{l}\text { Xmin }=1 \text { Xmax }=50 \\
\text { Ymin=1 Ymax }=50\end{array}$ \\
\hline End points for System2 & $\begin{array}{l}\text { Xmin }=51 \text { Xmax }=100 \\
\text { Ymin }=1 \text { Ymax }=50\end{array}$ \\
\hline End points for System3 & $\begin{array}{l}\text { Xmin=101 Xmax=150 } \\
\text { Ymin }=51 \text { Ymax }=100\end{array}$ \\
\hline End points for System4 & $\begin{array}{l}\text { Xmin=151 Xmax }=200 \\
\text { Ymin }=51 \text { Ymax }=100\end{array}$ \\
\hline End points for System5 & $\begin{array}{l}\text { Xmin=151 Xmax }=200 \\
\text { Ymin }=51 \text { Ymax }=100\end{array}$ \\
\hline $\begin{array}{l}\text { Number of Detection points } \\
\text { for System1 }\end{array}$ & 10 \\
\hline $\begin{array}{l}\text { Number of Detection points } \\
\text { for System2 }\end{array}$ & 10 \\
\hline $\begin{array}{l}\text { Number of Detection points } \\
\text { for System3 }\end{array}$ & 5 \\
\hline $\begin{array}{l}\text { Number of Detection points } \\
\text { for System4 }\end{array}$ & 10 \\
\hline $\begin{array}{l}\text { Number of Detection points } \\
\text { for System5 }\end{array}$ & 5 \\
\hline
\end{tabular}

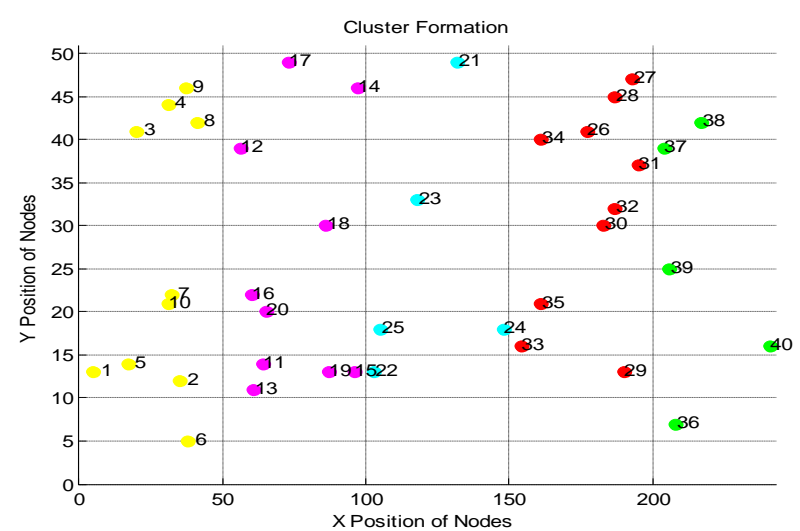

Fig. 21: Multi System Network 
Fig. 21 shows multi System network. As shown the fig System1 has the detection points \{Detection point 1, Detection point 2, Detection point 3, Detection point 4, Detection point 5 , Detection point 6 , Detection point 7 , Detection point 8, Detection point 9, Detection point 10$\}$. System 2 detection points which are between Detection point 11 till Detection point 20, System3 has the detection points between Detection point 21 to Detection point 25 and finally System4 has the detection points between Detection point 26 to Detection point 30.

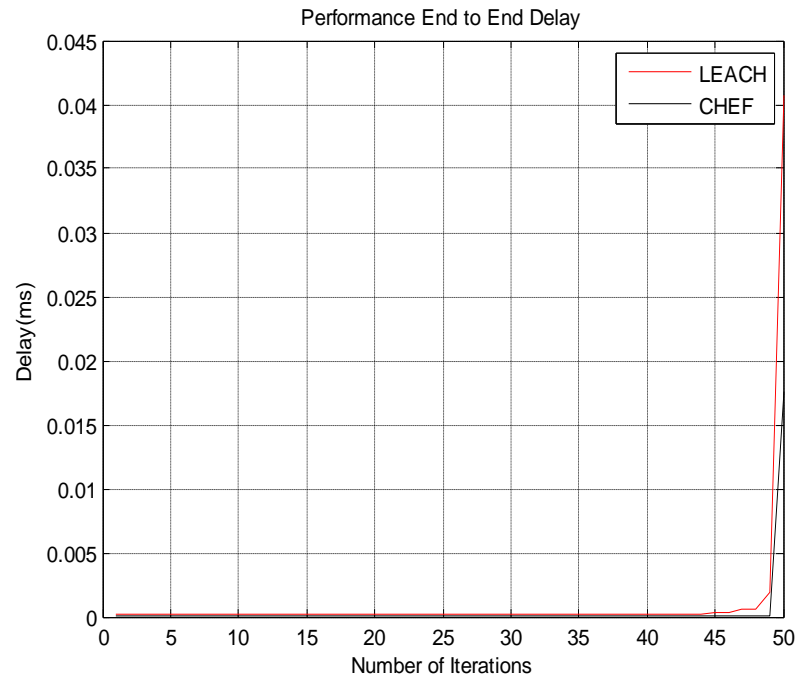

Fig 22: Delay Comparison

Fig. 22 shows the delay comparison between LEACH and CHEF. As shown in the fig. 22 CHEF method has low delay as compared to LEACH method. End to End Delay is measured as the time taken for the control packet to go from Source Detection point to Destination Detection point and vice versa.

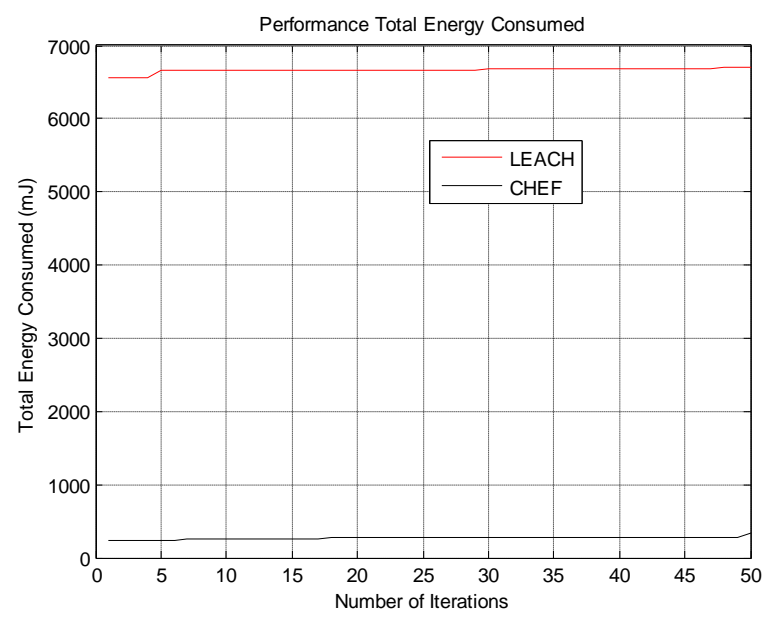

Fig. 23: Total Energy Consumption

Fig. 23 shows the total energy consumption measured for both CHEF and LEACH algorithm. As shown in the fig. 23 the CHEF method has the lowest energy consumption as compared to LEACH method. The energy consumption for CHEF is below $1000 \mathrm{~J}$ as compared to $\mathrm{LEACH}$ which is above $6000 \mathrm{~J}$.

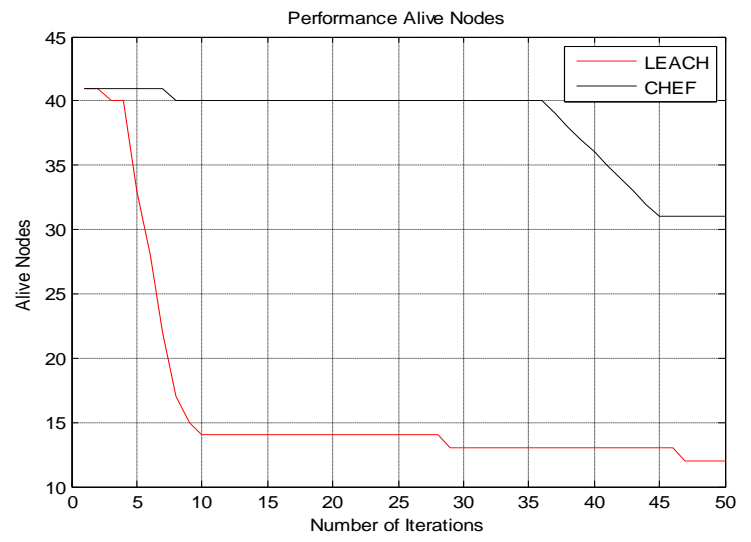

Fig 24: Non-Hole Detection points Comparison

Fig 24 shows the Non-Hole detection points comparison. As shown in the Fig 24 LEACH has less Non-Hole detection points as compared by CHEF. For LEACH method there are 8 detection points and then CHEF has the 32 Non-Hole detection points at the end of 50 iterations.

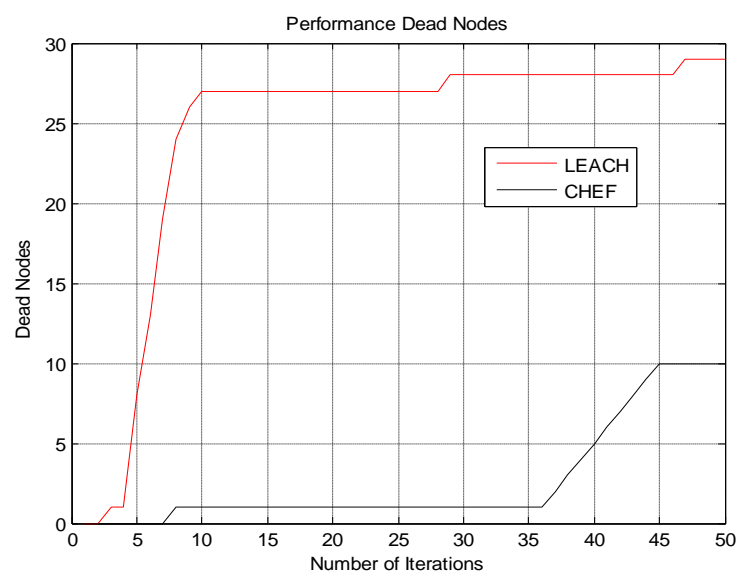

Fig. 25: Hole Detection points Comparison

Fig. 25 shows the comparison of number of Hole detection points between LEACH and CHEF method. As shown in the Fig $25 \mathrm{CHEF}$ has lower Hole detection points as compared to LEACH method. At the end of 50 iterations LEACH has 29 Hole detection points and CHEF method has 10 Hole detection points.

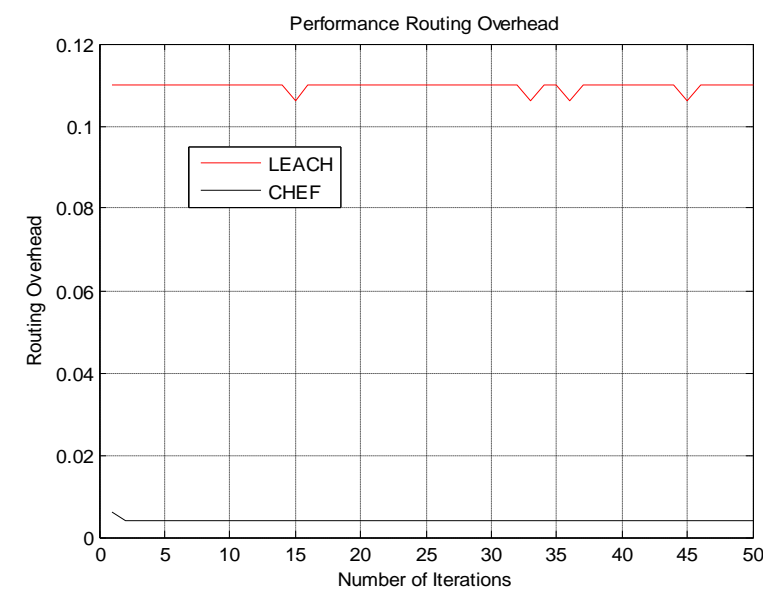

Fig. 26: Overhead Comparison 
Fig. 26 shows the overhead comparison. As shown in the fig. 26 the CHEF method has lower overhead ratio as compared to LEACH. For LEACH the overhead ratio is above 0.1 and for $\mathrm{CHEF}$ the overhead is lesser than 0.01. Hence CHEF behaves the best as compared to LEACH.

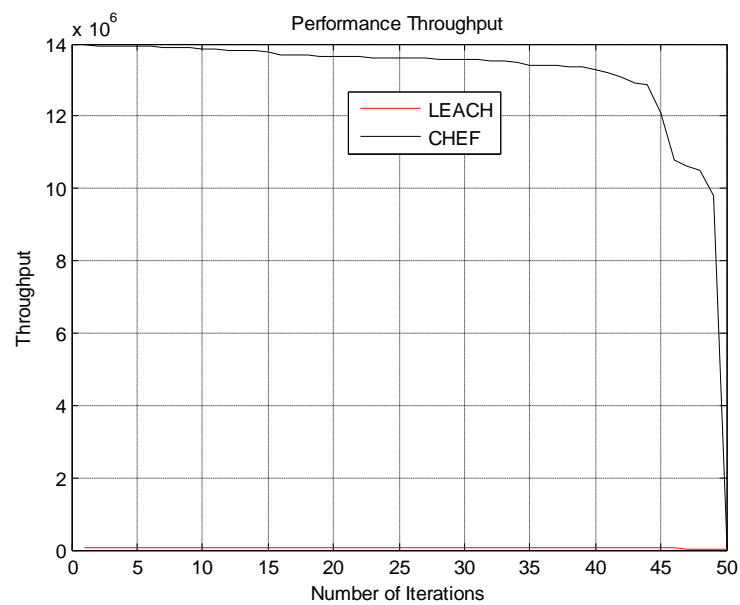

Fig. 27: Throughput Comparison

Fig. 27 shows the comparison of throughput. As shown in the fig. 27 the throughput of CHEF algorithm is way better as compared to LEACH method.

\section{CONCLUSION}

In this paper energy dissipation analysis along with NON-HOLE to HOLE ratio measure is described. The Classification of network is done into two kinds namely Single System Network and Multi System based network. LEACH and CHEF method are described in terms of algorithms. The algorithms are implemented using MATLAB. Simulation results of LEACH and CHEF are described with respect to network formation, first energy during formation of network, path between source detection point to destination detection point, updating the residual energy of detection points. The comparison is done between LEACH and CHEF with respect to delay, energy consumed, Non-Hole detection points, Hole detection points, overhead and throughput and it is proved that CHEF is the best. In future we will modify CHEF by improving the selection factor for group head and also the maintenance of path.

\section{REFERENCES}

1. Murugaanandam.S. and Ganapathy.V., "Reliability-based Cluster Head Selection Methodology using Fuzzy Logic for Performance Improvement in WSNs," in IEEE Access.

2. P. Zuo, T. Peng, K. You, W. Guo and W. Wang, "RSS-based Localization of Multiple Directional Sources with Unknown Transmit Powers and Orientations," in IEEE Access.

3. L. Spyrou, P. Chambers, M. Sellathurai and J. Thompson, "Tradeoffs in Detection and Localisation Performance for Mobile Sensor Scanning Strategies," 2019 Sensor Signal Processing for Defence Conference (SSPD), Brighton, United Kingdom, 2019, pp. 1-5.

4. A. R. Bhangwar et al., "WETRP: Weight based Energy \& Temperature aware Routing Protocol for Wireless Body Sensor Networks," in IEEE Access.

5. P. K. H. Kulkarni and P. Malathi Jesudason, "Multipath data transmission in WSN using exponential cat swarm and fuzzy optimisation," in IET Communications, vol. 13, no. 11, pp. 1685-1695, 1672019.

6. V. Potdar, A. Sharif, and E. Chang, "Wireless sensor networks: A survey," in Advanced Information Networking and Applications Workshops, International Conference on 2009, pp. 636-641.
7. V. Gungor and G. Hancke, "Industrial wireless sensor networks Challenges, design principles, and technical approaches," IEEE Transactions on Industrial Electronics, vol. 56, no. 10, pp. 4258-4265,October 2009.

8. "Wireless sensor networks: A survey," Computer Networks, vol. 38, no. 4, pp. 393-422, March 2002.

9. K. Romer and F. Mattern, "The design space of wireless sensor networks," IEEE Wireless Communications, vol. 11, no. 6, pp 54-61,December 2004.

10. Dietrich and F. Dressler, "On the lifetime of wireless sensor networks,"ACM Transactions on Sensor Networks, vol. 5, no. 1, pp. 1-39,February 2009.

11. M. Najimi, A. Ebrahimzadeh, S. Andargoli, and A. Fallahi, "Lifetime maximization in cognitive sensor networks based on the detection point selection,” IEEE Sensors Journal, vol. 14, no. 7, pp. 2376-2383, July 2014.

12. Y. Chen and Q. Zhao, "On the lifetime of wireless sensor networks," IEEE Communications Letters, vol. 9, no. 11, pp. 976-978, November 2005.

13. J. W. Jung and M. Weitnauer, "On using cooperative routing for lifetime optimization of multi-hop wireless sensor networks: Analysis and guidelines," IEEE Transactions on Communications, vol. 61, no. 8,pp. 3413-3423, August 2013.

14. C. Cassandras, T. Wang, and S. Pourazarm, "Optimal routing and energy allocation for lifetime maximization of wireless sensor networks with nonideal batteries," IEEE Transactions on Control of Network Systems, vol. 1, no. 1, pp. 86-98, March 2014.

15. B. Bejar Haro, S. Zazo, and D. Palomar, "Energy efficient collaborative beamforming in wireless sensor networks," IEEE Transactions on Signal Processing, vol. 62, no. 2, pp. 496-510, January 2014.

16. M. Bhardwaj and A. P. Chandrakasan, "Bounding the lifetime of sensor networks via optimal role assignments," in IEEE International Conference on Computer Communications (INFOCOM'02), vol. 3, NY,USA, June 2002, pp. 1587-1596.

17. S. Soro and W. Heinzelman, "Prolonging the lifetime of wireless sensor networks via unequal clustering," in IEEE International Parallel and Distributed Processing Symposium, Denver, CO, April 2005

18. B. C`arbunar, A. Grama, J. Vitek, and O. C arbunar, "Redundancy and coverage detection in sensor networks," ACM Transactions on Sensor Networks (TOSN), vol. 2, no. 1, pp. 94-128, February 2006.

19. X. Li and N. Xie, "Multi-Model Fusion Harvested Energy Prediction Method for Energy Harvesting WSN Detection point," 2018 IEEE International Conference on Electron Devices and Solid State Circuits (EDSSC), Shenzhen, 2018, pp. 1-2.

20. V. N. Vo, H. Tran, E. Uhlemann, Q. X. Truong, C. So-In and A Balador, "Reliable Communication Performance for Energy Harvesting Wireless Sensor Networks," 2019 IEEE 89th Vehicular Technology Conference (VTC2019-Spring), Kuala Lumpur, Malaysia, 2019, pp. 1-6.

21. S. Misra, S. K. Roy, A. Roy, M. S. Obaidat and A. Jha, "MEGAN: Multipurpose Energy-Efficient, Adaptable, and Low-Cost Wireless Sensor Detection point for the Internet of Things," in IEEE Systems Journal.

22. K. Chen, H. Gao, Z. Cai, Q. Chen and J. Li, "Distributed Energy-Adaptive Aggregation Scheduling with Coverage Guarantee For Battery-Free Wireless Sensor Networks," IEEE INFOCOM 2019 IEEE Conference on Computer Communications, Paris, France, 2019, pp. 1018-1026.

23. P. K. Sharma, Y. Jeong and J. H. Park, "EH-HL: Effective Communication Model by Integrated $\mathrm{EH}-\mathrm{WSN}$ and Hybrid LiFi/WiFi for IoT," in IEEE Internet of Things Journal, vol. 5, no. 3, pp. 1719-1726, June 2018

24. K. K. Pandey, B. Saud, B. Kumari and S. Biswas, "An energy efficient hierarchical clustering technique for wireless sensor network," 2016 Fourth International Conference on Parallel, Distributed and Grid Computing (PDGC), Waknaghat, 2016, pp. 544-549.

25. S. Sasirekha and S. Swamynathan, "Cluster-chain mobile agent routing algorithm for efficient data aggregation in wireless sensor network," in Journal of Communications and Networks, vol. 19, no. 4, pp. 392-401, August 2017. 
26. A. Mahboub, E. M. En-Naimi, M. Arioua, I. Ez-Zazi and A. El Oualkadi, "Multi-zonal approach clustering based on stable election protocol in heterogeneous wireless sensor networks," 2016 4th IEEE International Colloquium on Information Science and Technology (CiSt), Tangier, 2016, pp. 912-917.

27. S. Poolsanguan, C. So-In, K. Rujirakul and K. Udompongsuk, "An enhanced cluster head selection criterion of LEACH in wireless sensor networks," 2016 13th International Joint Conference on Computer Science and Software Engineering (JCSSE), Khon Kaen, 2016, pp. $1-7$.

28. N. Kumar and S. Kaur, "Performance evaluation of Distance based Angular Clustering Algorithm (DACA) using data aggregation for heterogeneous WSN," 2016 International Conference on Computation of Power, Energy Information and Commuincation (ICCPEIC), Chennai, 2016, pp. 097-101.

29. W. T. Gwavava and O. B. V. Ramanaiah, "YA-LEACH: Yet another LEACH for wireless sensor networks," 2015 International Conference on Information Processing (ICIP), Pune, 2015, pp. 96-101.

30. H. Y. Shwe and P. H. J. Chong, "Cluster-Based WSN Routing Protocol for Smart Buildings," 2015 IEEE 81st Vehicular Technology Conference (VTC Spring), Glasgow, 2015, pp. 1-5.

31. Faezeh Alavi, Kanapathippillai Cumanan, Zhiguo Ding, Alister G. Burr,"Beamforming Techniques for Non-Orthogonal Multiple Access in 5G Cellular Networks", IEEE Transactions on Vehicular Technology, 2018.

32. Irfan Ahmed, Hedi Khammari, Adnan Shahid, Ahmed Musa, Kwang Soon Kim, Eli De Poorter, Ingrid Moerman,"A Survey on Hybrid Beamforming Techniques in 5G: Architecture and System Model Perspectives",IEEE Communications Surveys \& Tutorials, 2018.

33. Devashish Arora, Meenakshi Rawat, "Comparative analysis of beamforming techniques for wideband signals", International Conference on Computing and Communication Technologies for Smart Nation (IC3TSN), 2017 Pages: 51 - 54.

34. Pogula Rakesh, S. Siva Priyanka,T. Kishore Kumar, "Performance evaluation of beamforming techniques for speech enhancement",Fourth International Conference on Signal Processing, Communication and Networking (ICSCN), Year: 2017, Pages: 1 - 5.

35. Spyridon Vassilaras,George C. Alexandropoulos,"Cooperative beamforming techniques for energy efficient IoT wireles communication", IEEE International Conference on Communications (ICC), Year: 2017,Pages: 1 - 6

36. Adnan Anwar Awan, Irfanullah, Shahid Khattak, Aqdas Naveed Malik, "Performance comparisons of fixed and adaptive beamforming techniques for $4 G$ smart antennas", International Conference on Communication, Computing and Digital Systems (C-CODE), Year: 2017 Pages: $17-20$

37. Anupama Senapati, Kaustabh Ghatak, Jibendu Sekhar Roy,"A Comparative Study of Adaptive Beamforming Techniques in Smart Antenna Using LMS Algorithm and Its Variants",International Conference on Computational Intelligence and Networks, Year: 2015 , Pages: $58-62$

38. Z. Han and H. Poor, "Lifetime improvement of wireless sensor networks by collaborative beamforming and cooperative transmission," in IEEE International Conference on Communications (ICC’07), Glasgow, June 2007, pp. 3954-3958.

39. Rong Du, Ayça Özçelikkale, Carlo Fischione, Ming Xiao, "Towards Immortal Wireless Sensor Networks by Optimal Energy Beamforming and Data Routing", IEEE Transactions on Wireless Communications, Year: 2018, ( Early Access ),Pages: $1-1$.

40. X. Deng, B. Wang, W. Liu, and L. Yang, "Sensor scheduling for multi-modal confident information coverage in sensor networks," IEEE Transactions on Parallel and Distributed Systems, vol. 26, no. 3, pp.902-913, March 2015.

41. C.-P. Chen, S. Mukhopadhyay, C.-L. Chuang, M.-Y. Liu, and J.-A Jiang, "Efficient coverage and connectivity preservation with load balance for wireless sensor networks," IEEE Sensors Journal, vol 15,no. 1, pp. 48-62, January 2015.

42. Q. Zhao and M. Gurusamy, "Lifetime maximization for connected target coverage in wireless sensor networks," IEEE/ACM Transactions on Networking, vol. 16, no. 6, pp. 1378-1391, December 2008.

43. Tajudeen O. Olasupo, Carlos E. Otero, "Framework for Optimizing Deployment of Wireless Sensor Networks", IEEE Transactions on Network and Service Management,Year-2018.

44. J. Matamoros and C. Antòn-Haro, "Opportunistic power allocation and sensor selection schemes for wireless sensor networks," IEEE Transactions on Wireless Communications, vol. 9, no. 2, pp. 534-539,February 2010.
45. Imad El Qachchach,Abdul Karim Yazbek, Oussama Habachi,Jean-Pierre Cances, Vahid Meghdadi, "New concatenated code schemes for data gathering in WSN's using rank metric codes", 2018 IEEE Wireless Communications and Networking Conference (WCNC), Year: 2018, Pages: $1-6$.

46. Kun Xie, Lele Wang, Xin Wang, Gaogang Xie, Jigang Wen,"Low Cost and High Accuracy Data Gathering in WSNs with Matrix Completion", IEEE Transactions on Mobile Computing,Year: 2018 Volume: 17, Issue: 7,Pages: $1595-1608$

47. Saurav Ghosh, Sanjoy Mondal,Utpal Biswas, "Enhanced PEGASIS using ant colony optimization for data gathering in WSN", 2016 International Conference on Information Communication and Embedded Systems (ICICES),Year: 2016,Pages: $1-6$

48. C. V. Phan, Y. Park, H. Choi, J. Cho, and J. G. Kim, "An energy efficient transmission strategy for wireless sensor networks," IEEE Transactions on Consumer Electronics, vol. 56, no. 2, pp 597-605,May 2010.

49. J. Kim, X. Lin, N. B. Shroff, and P. Sinha, "Minimizing delay and maximizing lifetime for wireless sensor networks with any cast," IEEE/ACM Transactions on Networking, vol. 18, no. 2, pp 515-528,April 2010.

50. F. Liu, C.-Y. Tsui, and Y. Zhang, "Joint routing and sleep scheduling for lifetime maximization of wireless sensor networks," IEEE Transactions on Wireless Communications, vol. 9, no. 7, pp. 2258-2267, July 2010 